\title{
TOMÁS DE AQUINO Y LA LEY NATURAL INTERPRETACIONES CRUZADAS DE SUMMA THEOLOGIAE I-II, 94, 2
}

Tomas de Aquino and the Natural Law

Summa Theologiae cross interpretations I-II, 94, 2

Thomas Aquino e la legge naturale

Interpretazioni incrociate di Summa Theologiae I-II, 94, 2

Carlos Massini-Correas ${ }^{1}$

Para citar este artículo:

Massini-Correas, C. (2020). “Tomás de Aquino y la ley natural. Interpretaciones cruzadas de Summa Theologiae I-II, 94, 2". Prudentia Iuris, N. Aniversario, pp. 63-77.

DOI: https://doi.org/10.46553/prudentia.aniversario.2020.pp.63-77

Resumen: El texto central del iusnaturalismo occidental es el de la Summa Theologiae, de Tomás de Aquino I-II, q. 94. a.2, en el que el Aquinate hace referencia a tres tipos de realidades: la naturaleza humana, las inclinaciones naturales y los bienes humanos. En general, se ha priorizado uno de estos tipos al momento de estudiar las vías de conocimiento de la ley natural, arrojando a los demás autores al rincón de los errores, imprecisiones y hasta maldades. Este trabajo sostiene la hipótesis de que el estudio de la naturaleza, las inclinaciones y los bienes como fuentes de la normatividad natural no es necesariamente excluyente, y que un análisis atento y desapasionado conduce a la conclusión de que se trata de vías diversas pero complemen-

1 Doctor en Ciencias Jurídicas y Sociales y en Filosofía. Catedrático de Filosofía Jurídica en la Universidad de Mendoza, Mendoza, Argentina. Profesor en la Maestría de Filosofía del Derecho en la Universidsad de Buenos Aires, Buenos Aires, Argentina. Y en el Doctorado en Ciencias Jurídicas en la Universidad de Mendoza, Mendoza, Argentina. Investigador Superior en Dirección de Investigaciones de la Universidad de Mendoza, Mendoza, Argentina. Correo electrónico: carlos.massini@um.edu.ar 
tarias, y que un estudio desde la sinergia de estas fuentes de conocimiento conduce a soluciones más ricas y explicativas que las que alcanzan quienes las ven como alternativas excluyentes.

Palabras clave: Tomás de Aquino; Summa Theologiae; Ley natural; Vías de conocimiento; Naturaleza humana.

Abstract: The central text of western natural law is that of the Summa Theologiae, of Thomas Aquinas I-II, q. 94. a.2, in which the Aquinate refers to three types of realities: human nature, natural inclinations and human goods. In general, one of these types has been prioritized when studying the ways of knowing Natural Law, throwing other authors into the corner of errors, inaccuracies and even evil. This work supports the hypothesis that the study of nature, inclinations and goods as sources of natural regulations is not necessarily exclusive, and that a careful and dispassionate analysis leads to the conclusion that these are diverse but complementary ways, and that a study from the synergy of these sources of knowledge leads to richer and more explanatory solutions than those achieved by those who see them as exclusive alternatives.

Keywords: Thomas Aquinas; Summa Theologiae; Natural Law; Ways of knowing; Human nature.

Sommario: Il testo centrale della legge naturale occidentale è quello della Summa Theologiae di Tomasso di Aquino I-II, q. 94. a.2, in cui l'Aquinate si riferisce a tre tipi di realtà: natura umana, inclinazioni naturali e beni umani. In generale, a uno di questi tipi è stata data la priorità quando si studia il modo di intendere la legge naturale, gettando gli altri autori nell'angolo di errori, imprecisioni e persino mali. Questo testo supporta l'ipotesi che lo studio della natura, delle inclinazioni e dei beni come fonti di normatività naturale non sia necessariamente esclusivo, e che un'analisi attenta e spassionata porti alla conclusione che si tratta di percorsi diversi ma complementari e che uno studio dalla sinergia di queste fonti di conoscenza porta a soluzioni più ricche ed esplicative di quelle raggiunte da chi le vede come alternative esclusive.

Parole chiave: Tomasso d'Aquino; Summa Theologiae; Legge naturale; Modi di conoscere; Natura umana. 


\section{El realismo ético y la tradición clásica}

Si bien el realismo ético-jurídico tiene varias concreciones y modalidades, algunas de ellas de factura típicamente contemporánea, resulta indudable que la versión paradigmática de esa orientación es la que se gestó en el ámbito del pensamiento clásico, en especial de lo que Isaiah Berlin

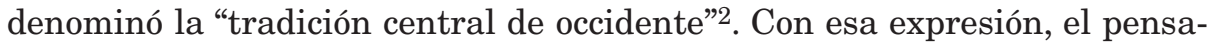
dor liberal designó la línea de pensamiento ético (moral personal, política, derecho, economía), que tiene su origen en el pensamiento ateniense de los siglos V y IV a.C., principalmente en las ideas de Sócrates, Platón y Aristóteles; tuvo sus representantes en el pensamiento romano, en especial en el de Cicerón; alcanzó un hito fundamental en el sistema de Tomás de Aquino; tuvo algunos desacuerdos con los nominalistas del siglo XIV ${ }^{3}$ y continuó su andadura con los escolásticos españoles del Siglo de Oro, así como con los sucesivos neo-escolasticismos y conservatismos, v. gr. el de Edmund Burke, que ha conocido el pensamiento occidental ${ }^{4}$.

Pero también en el marco de las ideas contemporáneas han surgido propuestas de revalorización de la ética clásica, sobre todo por parte de quienes siguen las ideas de Tomás de Aquino. Entre los autores que han encarado estas propuestas en los últimos años, tanto en el ámbito de la ética general, como de la filosofía jurídica, económica y política, cabe destacar a Antonio Millán-Puelles y Javier Hervada, en España; Heinrich Rommen, Josef Pieper y Robert Spaemann, en Alemania; Jacques Leclerc, Michel Villey y Georges Kalinowski, en Francia; Ralph McInerny, Vernon Bourke, Germain Grisez, Alasdair MacIntyre, Henry Veatch y Jean Porter, en los Estados Unidos; Giuseppe Graneris, Francesco Olgiati, Francesco D’Agostino y Francesco Viola, en Italia; Martin Rhonheimer, en Suiza; y John Finnis y John Haldane en Gran Bretaña, así como varios más en otros países.

Todos estos autores y varios otros se consideran a sí mismos, piensan y escriben como participando de una tradición común de pensamiento e investigación. En realidad, las cosas no pueden ser de otra manera, ya que cualquier empresa intelectual exitosa ha de realizarse inexorablemente en el marco de alguna tradición. Escribe en este punto Giuseppe Abbà que "ningún filósofo comienza a pesar desde cero; antes bien, se encuentra en-

2 Berlin, I. (1995). "The Crooked Timber of Humanity: Chapters in the History of Ideas”. Cit. por George, R. P. Making Men Moral. Civil Liberties and Public Morality. Oxford. Clarendon Press, 19. 221-250.

3 Véase: Villey, M. (1962). Lecons d'histoire de la philosophie du droit. Paris. Dalloz,

4 Véase: Hervada, J. (1987). Historia de la ciencia del Derecho Natural. Pamplona. EUNSA, passim. 
frentado a los problemas filosóficos que resultan planteados por la lectura que él ha hecho de las obras de otros filósofos [...]. Esto, que vale para un filósofo individual, vale también para un conjunto de filósofos que adoptan, en tiempos diversos, la misma figura de filosofía moral elaborada por el fundador de una estirpe o escuela filosófica. Continuando su desarrollo, defendiéndola, corrigiéndola, modificándola bajo la presión de nuevos problemas, de nuevas objeciones, de nuevas críticas, de nuevas figuras, ellos dan vida a lo que se llama una tradición de investigación"5.

Ahora bien, la tradición de investigación que corresponde al pensamiento clásico tiene una serie de notas que la destacan en el contexto de las diferentes tradiciones de especulación: ante todo, se trata de una tradición enormemente extensa, que abarca desde las enseñanzas orales de Sócrates hasta las ideas difundidas por autores estrictamente contemporáneos, es decir, más de veinticinco siglos ininterrumpidos de pensamiento; en segundo lugar, es una tradición ética que se encuentra enmarcada en un contexto filosófico completo, es decir, que comprende una metafísica, una teología (la cristiana y, en especial, la católica), una antropología filosófica, una epistemología, una lógica, una filosofía de la historia y así sucesivamente; en tercer lugar, se está frente a una empresa intelectual-moral especialmente intensa, en el sentido de que no sólo tiene una extensión temporal destacada, sino también que ha abordado y discutido en su seno prácticamente todos los problemas que se plantean al pensamiento ético y lo ha hecho con una intensidad y una acribia especialmente destacables; y finalmente, en cuarto lugar, se trata de un proyecto explicativo y propositivo que ha sido contrastado una enorme cantidad de veces con la experiencia concreta de las cosas humanas y en especial humano-sociales, y que ha debatido acerca de los problemas éticos que se han planteado en las diferentes etapas de la historia y con las diversas doctrinas competitivas que han aparecido a lo largo del tiempo.

En esta tradición, la principal línea de explicitación y defensa del realismo ético se denomina teoría de la ley natural, y ha sido objeto de diferentes desarrollos y modalizaciones a lo largo de los más de veinticinco siglos que ha durado su andadura en la cultura de occidente. En un relevante artículo publicado en la Stanford Encyclopedia of Philosophy, el profesor

5 Abbà, G. (1996). Quale impostazione per la filosofia morale? Roma. LAS, 27. Acerca de la noción de "tradición" en la ética, véase: Boyle, J. (1994). "Natural Law and the Ethics of Tradition”. En AA. VV., Natural Law Theory: Contemporary Essays. Ed. R.P. George. Oxford. Clarendon Press, 3-30 y MacIntyre, A. (1988). Whose Justice? Which Rationality? London. Duckworth, 12; sobre las ideas de MacIntyre en este punto, véase: Mauri, M. (1997). "Autoridad y tradición". En AA. VV., Modernidad y tradición. Una reflexión ética sobre la sociedad contemporánea. Barcelona. EDIT-EURO, 7-21. 
de Georgetown, Mark Murphy, escribe, respecto de esta teoría, que "algunos escritores usan este término [natural law theory] en un sentido tan amplio que cualquier teoría moral que sea una versión del realismo moral -es decir, cualquier teoría moral que sostenga que algunas afirmaciones morales positivas son literalmente verdaderas $-[. .$.$] cuenta como un punto de vista$ de ley natural"6.

Pero más adelante reconoce que es posible usar la locución en un sentido más estricto, de modo que no toda forma de realismo ético pueda reducirse a una teoría de la ley natural, con lo que se plantea la cuestión de cuándo precisamente se está frente a una teoría de la ley natural y no frente a otras formas de realismo práctico-moral. Y sobre esto sostiene que existe una forma ideal de proceder en este punto, "una que tome como su punto de partida el papel central que juega la teoría moral de Tomás de Aquino en la tradición de la ley natural. Si alguna teoría -concluye- es una teoría de la ley natural, esa es la de Tomás de Aquino"7; ella conformaría de este modo el significado focal de "teoría de la ley natural" y a ella misma habría que referirse especialmente para desarrollarla.

En adelante se seguirá la sugerencia del profesor norteamericano y se adoptará la versión aquiniana de la teoría de la ley natural como caso central y paradigmático de esa teoría, de modo tal que las doctrinas en análisis se considerarán de ley natural en la medida en que se asemejen o asimilen a la desarrollada por Tomás de Aquino. Es más, el estudio se centrará aquí principalmente en un texto clave del corpus tomista: el que corresponde a la Summa Theologiae I-II, q. 94, a. 2, que es el que sintetiza de mejor manera la problemática y el que ha sido objeto de más debates y mayores análisis. Una vez adoptada esta perspectiva de estudio, conviene esclarecer especialmente el sentido en que se usará la locución "ley natural" en lo sucesivo. En este punto, John Finnis precisa que "el término 'ley' en la frase 'ley natural' se refiere a ciertos estándares de elección correcta, estándares que son normativos (esto es, racionalmente directivos y 'obligatorios') porque son verdaderos y porque elegir de otro modo que de acuerdo con ellos resulta irrazonable" .

Y respecto al término "natural" y sus relacionados "por naturaleza", "de acuerdo con la naturaleza" y "de naturaleza", este mismo autor sostiene que ese término "significa alguna, o algunas de las siguientes afirmaciones:

6 Murphy, M. "The Natural Law Tradition in Ethics". The Stanford Encyclopedia of Philosophy (Winter 2011 Edition). Edward N. Zalta (ed.), https://plato.stanford.edu/archives/ win2011/entries/natural-law-ethics/.

7 Ibídem.

8 Finnis, J. "Natural Law". En Collected Essays-Volume I-Reason in Action. Oxford. Oxford University Press, 200. 
(a) que los estándares relevantes (principios y normas) no son 'positivos', es decir, que son directivos y previos a cualquier posición a través de una decisión individual, o elección de un grupo, o convención; (b) que los estándares relevantes son 'superiores' a las leyes positivas, convenciones y prácticas, es decir, proveen las premisas para la evaluación crítica y aprobación o repulsa justificada o desobediencia a esas leyes, convenciones o prácticas; (c) que los estándares relevantes conforman los requerimientos más demandantes de la razón crítica y que son objetivos, en el sentido de que una persona que deje de aceptarlos como estándares de juicio estará en el error; y (d) que la adhesión a esos estándares relevantes tiende sistemáticamente a promover el florecimiento humano, la realización de las comunidades y los individuos humanos"9.

\section{La ley natural en la tradición tomista}

Una vez precisado el sentido de la locución "ley natural", corresponde sintetizar en algunos puntos específicos el núcleo de la doctrina de Tomás de Aquino acerca de esta problemática ${ }^{10}$, limitándonos, en principio, al texto mencionado de la Summa Theologiae. En primer lugar, conviene decir que "ley natural", en clave tomista, es un conjunto de principios normativos o proposiciones práctico-normativas primeras que dirigen la conducta humana hacia su bien-perfección integral, y, en cuanto tal, ese conjunto debe ser distinguido de los estudios filosóficos o científicos que tienen como objeto a esos principios. Estos últimos estudios se denominan "teoría (o filosofía) de la ley natural" y, en su conjunto, forman una amplia orientación que se denomina genéricamente "iusnaturalismo", pero no son propiamente la "ley natural", sino su estudio sistemático a nivel filosófico ${ }^{11}$.

Ahora bien, en estos principios es posible distinguir uno absolutamente primero, que puede denominarse "primer principio práctico" y cuya expresión tomista es "el bien ha de hacerse y el mal evitarse"12. Este principio,

9 Ídem, 200-201.

10 En este punto véase: Budziszewski, J. (2014). Commentary on Thomas Aquinas's Treatise on Law. New York. Cambridge University Press, 228 y sigs.; asimismo, véase: AA. VV. (2002). The Ethics of Aquinas. Ed. S. J. Pope. Washington DC. Georgetown University Press, passim.

11 Véase: Finnis, J. (2008). "La tradición de la ley natural”. En Ideas y Derecho, Nº 6. Buenos Aires, 223.

12 Tomás de Aquino. Summa Theologiae (en adelante, ST), I-II, q. 94, a. 2. Sobre los debates existentes acerca del sentido de este principio, véase: May, W. E. (2004). "Contemporary Perspectives on Thomistic Natural Law" y Long, S. A. "Natural Law or Autonomous Practical Reason". En AA. VV. Thomas Aquinas and the Natural Law Tradition. Contemporary Perspec- 
siempre según Tomás de Aquino, cumple en el orden práctico la misma función que el principio de no-contradicción cumple en el orden especulativo, es decir, sirve de estructura fundamental de todo pensamiento práctico-normativo. En este punto, Ross Armstrong ha escrito que "el bien ha de hacerse y el mal evitarse' tiene el estatus de un principio directivo y es de acuerdo con este principio que todos los preceptos de la ley natural deben ser formulados [...]; este principio tiene un rol en la ley natural que lo hace distinto de todos los restantes preceptos: es un principio en el cual están fundadas todas las otras prescripciones de la ley natural"13. Puede decirse, entonces, que ese primer principio práctico es como la contextura básica de las normas más determinadas, que prescriben la realización de bienes humanos más específicos en contextos más delimitados.

$\mathrm{Y}$ estas normas más determinadas resultan necesarias toda vez que el hombre no actúa en principio por el bien en general, considerado en abstracto, sino por bienes determinados y concretos, que son los que resultan proporcionados a la voluntad humana, que no está ordenada -al menos en el aquende la muerte- al bien absoluto, sino a bienes de algún modo limitados y específicos. Escriben en este punto Grisez, Finnis y Boyle que "la diversidad de los bienes básicos no es ni un hecho contingente de la psicología humana ni un accidente de la historia [...]. Más bien, al ser aspectos de la realización de las personas, esos bienes se corresponden con las complejidades inherentes a la naturaleza humana"14. Y por ello, la vida, la procreación, la salud y la integridad física son bienes humanos básicos en razón de que el hombre es un ser animado; el conocimiento y la apreciación de la belleza son bienes humanos porque el hombre es cognoscente y racional; la amistad, la vida política y las demás formas de vida social son bienes humanos porque el hombre es un ser naturalmente social, y así sucesivamente ${ }^{15}$.

Pero además, el Aquinate hace referencia en este punto no sólo a la naturaleza humana en sí misma ("La ley natural es consecuencia de la naturaleza humana"16) y a los bienes humanos ("Todos los demás preceptos [...] tendrán carácter de preceptos de ley natural en cuanto la razón prácti-

tives. Ed. J. Goyette et alii. Washington DC. The Catholic University of America Press, 113-156 y $165-193$.

13 Armstrong, R. (1966). Primary and Secondary Precepts in Thomistic Natural Law Teaching. The Hague. Martinus Nijhoff, 40. En este texto, la expresión "fundadas" debería entenderse en el sentido de que los restantes principios práctico-normativos participan de su estructura formal.

14 Grisez, G.; Boyle, J. y Finnis, J. (1987). "Practical Principles, Moral Truth and Ultimate Ends". En The American Journal of Jurisprudence, N $^{\circ} 32$. Notre Dame, 107.

15 Ibídem, 107-108.

$16 S T$, I-II, q. 94, a. $2,2^{\text {a }}$ objeción. 
ca los aprehenda como [ordenados a los] bienes humanos"17), sino también a las inclinaciones que tienden a esos bienes ("todas las cosas hacia las que el hombre siente inclinación natural, son aprehendidas naturalmente como buenas y consecuentemente como que han de realizarse"18). Ahora bien, paralelamente a estas tres referencias, diferentes grupos de autores que se asumen como tomistas han defendido diversas doctrinas acerca de la prioridad o posterioridad de cada una de esas realidades al momento de la determinación epistémica de los contenidos de la ley natural. Y, además, en varios casos, se han considerado esas distintas referencias de modo excluyente, es decir, estudiándolas como si fuera necesario escoger entre una $\mathrm{u}$ otra de ellas de modo absoluto, descartando o relegando tajantemente a las restantes.

\section{Las interpretaciones principales}

El primero de estos grupos es el que puede llamarse "derivacionista", ya que los autores que lo integran sostienen que la materia o el contenido de las normas de la ley natural se conoce solo por derivación a partir de las dimensiones constitutivas de la naturaleza humana. "El bien concreto para el hombre -afirma Frederick Copleston- solo puede ser conocido por una reflexión sobre la naturaleza humana, tal como se la conoce por la experiencia"19. Y por su parte, Anthony Lisska sostiene que "la concepción del bien, [...] tal como es discutida por Aristóteles y Tomás de Aquino, está derivada de la esencia o naturaleza humana. El concepto aristotélico de esencia humana [...] resulta mejor dilucidado en términos de un conjunto de propiedades disposicionales. En Aristóteles y el Aquinate los bienes humanos, en cuanto fines, están conectados con la estructura de la persona humana"20. Por lo tanto, según estos autores y los que piensan como ellos, el modo adecuado de conocer el contenido de las normas de la ley natural es el de aprehender previamente las dimensiones centrales del modo de ser del hombre y luego inferir de allí cuáles son los bienes humanos y las normas y principios que a ellos se ordenan.

Por otra parte, un segundo grupo de autores, que puede llamarse "inclinacionista", sostiene que esos contenidos se conocen a partir de las inclinaciones naturales del hombre, que Tomás de Aquino reduce a tres: (i) inclinación a la conservación de la vida e integridad física, (ii) a la procreación y

17 Ibídem.

18 Ibídem.

19 Copleston, F.C. (1991). Aquinas. London. Penguin, 232.

20 Lisska, A. (1995). Aquinas's Theory of Natural Law. Oxford. Clarendon Press, 143. 
educación de la prole y (iii) a la vida social y el conocimiento de la verdad ${ }^{21}$. Cada una de estas inclinaciones se corresponde con una de las dimensiones de la naturaleza humana: la vegetativa, la animada y la racional y, según estos intérpretes del Aquinate, estas inclinaciones resultarían la fuente privilegiada para el conocimiento de las normas de la ley natural. En ese sentido, Reginaldo Pizzorni afirma que "Santo Tomás determina el contenido del Derecho Natural en base a las tres inclinaciones esenciales del hombre" 22 .

Finalmente, otro conjunto de autores -el tercero, que puede denominarse "intuicionista"- coloca el punto de partida del conocimiento de los contenidos de las normas éticas naturales en la captación inmediata o intuitiva de las determinaciones centrales del bien humano o "bienes humanos básicos"23. Para estos pensadores, el orden del conocimiento de la ley natural no es idéntico al orden de la dependencia ontológica de sus contenidos normativos, ya que según un principio defendido por Tomás de Aquino -llamado por Finnis "principio epistemológico"-, el conocimiento de la naturaleza humana depende epistémicamente del de sus facultades o inclinaciones, facultades que, a su vez, se conocen por sus actividades, las que por su parte se especifican por sus objetos.

De este modo, v. gr., la naturaleza libre del hombre se conoce a través del ejercicio libre de la voluntad electiva, cuyo carácter se aprehende por medio del conocimiento de sus actividades autónomas, que solo pueden ejercerse en el ámbito de las realidades contingentes. En el caso del conocimiento de los contenidos de la ley natural, Finnis afirma que este se inicia por la aprehensión por auto-evidencia de los bienes humanos básicos, los que a su vez hacen posible aprehender las praxis humanas que los tienen por objeto, praxis que son el medio de conocer las potencias o facultades del hombre, facultades que indican las dimensiones de la naturaleza en las que arraigan y hacen posible percibir y discernir el concepto y los contenidos de la naturaleza humana ${ }^{24}$.

Finnis precisa, además, en este punto que "si cambiamos del modo epistemológico al ontológico, el mismo principio metodológico, en su aplicación a los seres humanos, presupone y por lo tanto implica que la bondad de todos

21 ST, I-II, q. 94, a. 2.

22 Pizzorni, R. (1974). "Il contenuto del diritto naturale secondo Tommaso d'Aquino". En AA. VV. San Tommaso e la filosofia del diritto oggi. Roma. Studi Tomistici, 210. Véase también: Composta, D. (1971). Natura e ragione. Studio sulle inclinazione naturali in rapporto all diritto naturale. Zürich. PAS-Verlag, 70-106.

23 En especial, véase: Finnis, J. (2011). Natural Law and Natural Rights. $2^{\mathrm{a}}$ ed. Oxford. Oxford University Press, 59-99.

24 Finnis, J. (2011). "Is and Ought in Aquinas". En Collected Essays-I-Reason in Action. Oxford. Oxford University Press, 147. 
los bienes humanos [...] está derivada de (i.e. depende de) la naturaleza, a la que, en razón de su bondad, esos bienes perfeccionan. Porque esos bienes -que en cuanto fines son las rationes de las normas o 'deberes' prácticos- no perfeccionarían esa naturaleza si ella fuera distinta de lo que es. Por lo tanto, el debe depende ontológicamente -y en este sentido seguramente puede decirse que está derivado- del es"25. Por lo tanto, queda en claro que este autor reconoce expresa, explícita e inequívocamente la dependencia, en el orden ontológico o metafísico, de los bienes humanos y de las normas que dirigen hacia ellos, respecto a los rasgos esenciales de la naturaleza humana ${ }^{26}$.

Ahora bien, como se expuso más arriba, gran parte de los pensadores mencionados precedentemente consideran que la vía cognitiva desarrollada por ellos resulta excluyente de las restantes, al menos para todos los efectos prácticos. Como se ha visto, no es el caso precisamente de John Finnis, pero esta exclusividad le ha sido reprochada, sin verdadero fundamento en los textos, por varios autores ${ }^{27}$. Pero si se realiza un análisis riguroso de los textos del Aquinate, queda en claro que las tres orientaciones mencionadas acerca de la fuente de los contenidos -o fundamento material- de la ley natural no deberían plantear de modo necesario alternativas excluyentes sino que, por el contrario, es posible que esas alternativas resulten complementarias y mutuamente enriquecedoras. Se trata, en realidad, de distintos puntos de vista o dimensiones, que priorizan diferentes aspectos de una realidad compleja y que pueden ser estructurados armónicamente y sin exclusiones tajantes. De hecho, en el texto del Aquinate al que remite Finnis (“[...] por los objetos conocemos los actos, por los actos las potencias y por las potencias la esencia [o naturaleza] del alma"28), queda en claro que entre los objetos de los actos humanos (es decir, los bienes humanos), los actos y las potencias que los realizan y la naturaleza en la que se fundamentan existe una sinergia que puede ser estudiada desde cada uno de sus elementos con un resultado enriquecedor y no excluyente ${ }^{29}$.

25 Ibídem.

26 Véase: Massini-Correas, C. I. (2014). "Sobre bienes humanos, naturaleza humana y ley natural. Reflexiones a partir de las ideas de Javier Hervada y John Finnis”. En Persona \& Derecho, No 70-71. Pamplona, 229-256.

27 Véase, entre varios otros: Hittinger, R. (1987). A Critique of the New Natural Law Theory. Notre Dame-Indiana. University of Notre Dame Press. Para una precisa refutación de estas críticas, véase: George, R. P. (2001). In Defense of Natural Law. Oxford. Oxford University Press, $15-91$.

28 Tomás de Aquino (1979). Comentario al libro Del Alma de Aristóteles, Lec. VI, n. 308. Se cita conforme a la traducción de M. C. Donadío de Gandolfi. Buenos Aires. Fundación Arché.

29 Véase, en este punto: Crowe, J. (2017). "Metaphysical Foundations of Natural Law Theories". En AA. VV. The Cambridge Companion to Natural Law Jurisprudence. Eds. G. Duke \& R.P. George. Cambridge-New York. Cambridge University Press, 103-129. 
Y esto es así toda vez que la perspectiva metafísica -llamada por Finnis "ontológica", aunque esta última terminología resulta discutible ${ }^{30}-$ se refiere a las estructuras de la realidad, fundamentalmente la humana, en cuanto su realización completa, perfección o excelencia dependen constitutivamente de la índole de esa realidad, ya que no es posible hablar de la perfección de alguna cosa sin conocer las estructuras fundamentales de esa cosa. Pero es también un dato de la realidad el que exista una inclinación o tendencia propia y congénita de toda realidad hacia su perfección propia, de modo tal que, para usar las palabras del Estagirita, "el bien es aquello a lo que todas las cosas tienden"31; en este caso, se está frente a una dimensión apetitiva o de aspiración, que se expresa en tendencias que se ordenan y procuran la realización plena de alguna de las dimensiones que son objeto de estudio del conocimiento metafísico. Esta dimensión tendencial es por su parte objeto de estudio principalmente de la antropología filosófica. Y finalmente, está la realización misma de esa tendencia, fundada en la estructura metafísica de su sujeto, que es el objeto de las inclinaciones naturales, y que radica en las diferentes dimensiones del bien del ente de que se trate; en el caso del ser humano y su actividad electiva, se trata del bien moral ${ }^{32}$, y su consideración, explicitación y prescripción es tarea propia de la ética.

Y esto es así, toda vez que en el modo propio del pensamiento clásico, el dinamismo moral del ser humano no tiene la estructura excluyente y univocista propia del pensamiento moderno, en el cual, tomando prestado para la ética el método de conocimiento propio de las ciencias exactas y naturales, las alternativas de verdadero-falso se dan de un modo categórico y absoluto, a la vez que simplista, con evidente contraste con el modo analógico de conocimiento que es el propio de la especulación clásica. Esto es así, toda vez que en el caso del pensamiento práctico, también se da la posibilidad de un abordaje múltiple, gradual o matizado propio del abordaje analógico ${ }^{33}, \mathrm{y}$ por lo tanto, resulta oportuna la exclusión de toda perspectiva univocista 0 absolutista de las realidades práctico-morales.

30 Acerca de la aparición y uso del término “ontológico", véase: Ferrater Mora, J. (1979). Diccionario de Filosofía. T. 3. Madrid. Alianza, 2421 y sigs. Allí el autor español atribuye la primacía en el uso de esa locución al filósofo alemán Rudolf Goclenius, en la página 16 de su libro Lexicon Philosophicum quo tanquam clave philosophiae fores aperiuntur, de 1613, aunque su uso fue establecido y difundido unos años después por Leibniz y Christian Wolff.

31 Aristóteles. Ética Nicomáquea, I, 1, 1094 a 2-3. En este punto, véase: AA. VV. (2006). The Blackwell Guide to Aristotle's Nicomachean Ethics. Malden-Oxford-Carlton. Ed. R. Kraut, Blackwell Publishing, 37-95.

32 Véase: González, A. M. (2009). La ética explorada. Pamplona. EUNSA, 13-34.

33 Véase: Massini-Correas, C. (2012). "Entre reductivismo y analogía. Sobre el punto de partida de la Filosofía del Derecho”. En Persona \& Derecho, Nº 67. Pamplona, 353-385. 
Por ello, un abordaje integral y diversificado del fenómeno ético-jurídico, que además se armonice con las explicaciones de Tomás de Aquino en el lugar estudiado, debería analizar tanto los aspectos teórico-estructurales, cuanto los apetitivo-tendenciales y los práctico-télicos, en la búsqueda de una explicación completa y matizada de la praxis humana. Porque esa praxis es la actividad de un sujeto dotado de una naturaleza, orientado tendencialmente a buscar y realizar su plenitud, la que se alcanza por medio de ciertas perfecciones que se denominan bienes. Pero a su vez, estos bienes son tales por naturaleza, se alcanzan por inclinación natural y adquirida (virtudes) y la naturaleza adquiere dimensión práctica cuando especifica los bienes que la hacen florecer y culminar. Se trata, por lo tanto, en el caso de la actividad humana, de una sinergia en la que cada uno de sus elementos contribuye a hacer posible la realización completa de la plenitud personal.

En resumen, es posible afirmar que, conforme al pensamiento de Tomás de Aquino, la doctrina del realismo ético que corresponde a la Tradición Central de Occidente se estructura en torno a las siguientes afirmaciones: (i) en el orden práctico-moral, la dirección de la conducta humana hacia el bien o perfección del hombre, hacia su acabamiento o realización, se realiza principalmente a través de leyes-normas, es decir, de proposiciones prácticas que ordenan la conducta humana hacia el bien humano común y personal; (ii) estas proposiciones tienen carácter racional no sólo porque son producto de la facultad racional, sino porque su contenido es una medida u orientación razonable del obrar humano hacia el bien ${ }^{34}$; en otros términos, el referente de estas proposiciones es una relación de adecuación entre una conducta y una concreción objetiva del bien humano, aunque en cada norma concreta existe cierta medida de elaboración humana (podría decirse de "construcción") que determina la vía precisa de la ordenación de la conducta hacia alguna dimensión del bien humano; (iii) el descubrimiento de esos bienes se realiza, en primer lugar, a partir de la aprehensión por evidencia del primer principio práctico (conocido con ayuda de la sindéresis) y de los preceptos normativos universales y generales que se siguen estructuralmente de él, pero también por referencia al fundamento ontológico de los bienes, radicado en las dimensiones centrales de la naturaleza humana y expresado en las inclinaciones naturales hacia los bienes humanos; (iv) la ley natural funciona como elemento preceptivo y crítico respecto de las leyes humanas positivas, jurídicas o no, que resultan correctas en la medida en que se adecuan a las exigencias de la ley natural; y, finalmente, (v) los bienes humanos y los preceptos de la ley natural que a ellos se ordenan proporcionan razones

34 Véase, acerca de la función de la noción de "bien" en la ética: Clavier, P. (2010). Qu'est-ce que le bien? Paris. Vrin, passim. 
para la acción humana correcta, es decir, fundamentan y justifican objetivamente el sentido ético de la acción humana ${ }^{35}$.

\section{Conclusión sumaria}

Luego de los desarrollos realizados, corresponde arribar a ciertas conclusiones precisivas, que es posible sintetizar en las siguientes: (i) el iusnaturalismo o teoría de la ley natural es una tradición de pensamiento que integra la filosofía práctico-moral de matriz clásica, que se ha desarrollado desde su nacimiento en la Grecia del siglo V a.C., y que aun en nuestros días participa activamente en los debates filosófico-jurídicos contemporáneos; (ii) uno de los núcleos principales, en rigor el principal, de esa tradición iusnaturalista está constituido por los textos de Tomás de Aquino referidos a la ley natural; por ello, para conocer la significación focal del concepto de ley natural, resulta conveniente remitirse a esos textos, y en especial al contenido en la I-II, q. 94, a. 2 de la Summa Theologiae, en el que se efectúa un sumario sintético de la doctrina aquinatense; (iii) en el locus mencionado, el Aquinate hace referencia a tres elementos integrantes del fenómeno de la ley natural: la naturaleza humana, las inclinaciones naturales y los bienes humanos; algunos intérpretes de ese texto han manifestado, a veces, una inclinación a comprender esos tres elementos de modo parcialista y excluyente, es decir, priorizando alguno de ellos con descarte y preterición de los demás; (iv) en el presente escrito se ha sostenido tentativamente la tesis contraria, es decir, la que sostiene la posibilidad de una explicación de la ley natural a través de una justificación conjunta y armónica que tenga en cuenta los tres elementos enunciados de un modo complementario; (v) en definitiva, lo que se procura en el presente estudio es una superación de cualquier visión parcialista de la ley natural y la propuesta de una explicación armónica, integral y plural, que pareciera ser la que mejor se armoniza con el espíritu y la sistemática de la exposición efectuada por el Aquinate en su Summa Theologiae.

\section{Bibliografía}

AA. VV. (2002). The Ethics of Aquinas. Ed. S. J. Pope. Washington DC. Georgetown University Press, passim.

Abbà, G. (1996). Quale impostazione per la filosofia morale? Roma. LAS, 27.

35 Véase, en este punto: Daguet, F. (2015). Du politique chez Thomas d'Aquin. Paris. Librairie Philosophique J. Vrin, 209-266. 
Aristóteles. Ética Nicomáquea, I, 1, 1094 a 2-3. En este punto, véase: AA.VV. (2006). The Blackwell Guide to Aristotle's Nicomachean Ethics. Malden-Oxford-Carlton. Ed. R. Kraut. Blackwell Publishing, 37-95.

Armstrong, R. (1966). Primary and Secondary Precepts in Thomistic Natural Law Teaching. The Hague. Martinus Nijhoff, p. 40.

Berlin, I. "The Crooked Timber of Humanity: Chapters in the History of Ideas". Cit. por George, R. P. (1995). Making Men Moral. Civil Liberties and Public Morality. Oxford. Clarendon Press, 19.

Boyle, J. "Natural Law and the Ethics of Tradition". En AA. VV. (1994). Natural Law Theory: Contemporary Essays. Ed. R. P. George. Oxford. Clarendon Press, 3-30.

Budziszewski, J. (2014). Commentary on Thomas Aquinas's Treatise on Law. New York. Cambridge University Press, 228 y sigs.

Clavier, P. (2010). Qu'est-ce que le bien? Paris. Vrin, passim.

Composta, D. (1971). Natura e ragione. Studio sulle inclinazione naturali in rapporto all diritto naturale. Zürich. PAS-Verlag, 70-106.

Copleston, F. C. (1991). Aquinas. London. Penguin, 232.

Crowe, J. "Metaphysical Foundations of Natural Law Theories". En AA. VV. (2017). The Cambridge Companion to Natural Law Jurisprudence. Eds. G. Duke \& R.P. George. Cambridge-New York. Cambridge University Press, 103-129.

Daguet, F. (2015). Du politique chez Thomas d'Aquin. Paris. Librairie Philosophique J. Vrin, 209-266.

Ferrater Mora, J. (1979). Diccionario de Filosofía. T. 3. Madrid. Alianza, 2421 y sigs.

Finnis, J. (2011). "Is and Ought in Aquinas". En Collected Essays-I-Reason in Action. Oxford. Oxford University Press, 147.

Finnis, J. (2008). "La tradición de la ley natural”. En Ideas y Derecho, № 6. Buenos Aires, 223.

Finnis, J. "Natural Law". En Collected Essays-Volume I-Reason in Action. Oxford. Oxford University Press, 200.

Finnis, J. (2011). Natural Law and Natural Rights. $2^{\text {a }}$ ed. Oxford. Oxford University Press, 59-99.

George, R. P. (2001). In Defense of Natural Law. Oxford. Oxford. University Press, 15-91.

González, A. M. (2009). La ética explorada. Pamplona. EUNSA, 13-34.

Grisez, G.; Boyle, J. y Finnis, J. (1987). "Practical Principles, Moral Truth and Ultimate Ends". En The American Journal of Jurisprudence, No 32. Notre Dame, 107.

Hervada, J. (1987). Historia de la ciencia del Derecho Natural. Pamplona. EUNSA, passim.

Hittinger, R. (1987). A Critique of the New Natural Law Theory. Notre Dame-Indiana. University of Notre Dame Press.

Lisska, A. (1995). Aquinas's Theory of Natural Law. Oxford. Clarendon Press, 143.

MacIntyre, A. (1988). Whose Justice? Which Rationality? London. Duckworth, 12.

Massini-Correas, C. I. (2012). "Entre reductivismo y analogía. Sobre el punto de partida de la Filosofía del Derecho". En Persona \& Derecho, № 67. Pamplona, 353385 . 
Massini-Correas, C. I. (2014). "Sobre bienes humanos, naturaleza humana y ley natural. Reflexiones a partir de las ideas de Javier Hervada y John Finnis”. En Persona \& Derecho, No 70-71. Pamplona, 229-256.

Mauri, M. (1997). “Autoridad y tradición”. En AA.VV. Modernidad y tradición. Una reflexión ética sobre la sociedad contemporánea. Barcelona. EDIT-EURO, 7-21.

May, W. E. "Contemporary Perspectives on Thomistic Natural Law" y Long, S. A. "Natural Law or Autonomous Practical Reason". Ambos en AA. VV. (2004). Thomas Aquinas and the Natural Law Tradition. Contemporary Perspectives. Ed. J. Goyette et alii. Washington DC. The Catholic University of America Press, 113156 y 165-193.

Murphy, M. (winter 2011 edition). “The Natural Law Tradition in Ethics”. The Stanford Encyclopedia of Philosophy. Edward N. Zalta (ed.), https://plato.stanford. edu/archives/win2011/entries/natural-law-ethics/.

Pizzorni, R. (1974). "Il contenuto del diritto naturale secondo Tommaso d'Aquino". En AA. VV. San Tommaso e la filosofia del diritto oggi. Roma. Studi Tomistici, 210 .

Tomás de Aquino. Comentario al libro Del Alma de Aristóteles, Lec. VI, n. 308. Conforme a la traducción de M. C. Donadío de Gandolfi (1979). Buenos Aires. Fundación Arché.

Tomás de Aquino. Summa Theologiae, I-II, q. 94, a. 2.

Villey, M. (1962). Lecons d'histoire de la philosophie du droit. Paris. Dalloz, 221-250. 